\title{
Bone Osteosarcoma
}

National Cancer Institute

\section{Source}

National Cancer Institute. Bone Osteosarcoma. NCI Thesaurus. Code C53707.

A usually aggressive malignant bone-forming mesenchymal neoplasm arising from the bone. It may arise de novo or from a pre-existing lesion of the bone. Pain and a palpable mass are the most frequent clinical sign and symptom. It may spread to other anatomic sites, particularly the lungs. 